\title{
Failure Induced by Instability in Structural Composites under Longitudinal Compression
}

\author{
Alfredo R. de Faria, Ragnar Larsson* \\ Department of Mechanical Engineering, Instituto Tecnológico de Aeronáutica, São José dos Campos, SP/Brazil
E-mail: arfaria@ @ita.br, ragnar.larsson@ @ chalmers.se
*Department of Industrial and Materials Science, Chalmers University of Technology, Göteborg, Sweden
}

\begin{abstract}
The objective of this work is to develop a damage propagation model applicable to the study of failure mechanism in composites induced by longitudinal compression. The damage propagation model shall be based on an energy principle, quantified by the critical energy release rate associated to matrix cracking, and on the study of the kinematics of the equilibrium of a representative volume of a ply material within the damaged region of the laminate. It is expected that the model proposed will be implemented within the context of the finite element technology in order to expand its usefulness to realistic situations where multiple ply laminates are used. The model to be proposed shall capture the onset of formation of kink-bands and subsequently its propagation. It is formulated within the context of an energy based approach that considers the critical energy release rate of the matrix in the failed elements of the mesh. A damage parameter that ranges from 0 (intact) to 1 (fully damaged) is used that relates relative shear strains in the matrix induced by loss of stability of a representative volume. The damage models developed will be useful to realistically predict failure due to kink-band formation and propagation mechanism. The results obtained will serve as the starting point for larger collaborative projects involving strain rate effects and damage tolerant composites.
\end{abstract}

Keywords: Composites, damage tolerance, stress analysis

\section{Introduction}

The most prominent failure mode in fiber reinforced composites subject to longitudinal compressive loadings is kink-band formation. Rosen [1] was the first researcher to propose a model to describe this type of failure in fiber reinforced composites. However, Rosen's model, and a series of subsequent models based on his model, predicted unrealistic high strengths. It was only a few years later that Argon [2] proposed an improved model that substantially reconciled experimental and theoretical values of longitudinal compressive strengths. The great contribution from Argon was the consideration of initial local fiber misalignment that facilitated initialization of fiber instability followed by matrix shearing. Although Argon's model did not consider additional fiber rotation due to the initial misalignment it paved the way for other researchers to propose more accurate models [3, 4].

Failure by kink-band formation is today recognized to be a consequence of microbuckling and kinking, which are induced by matrix yielding or cracking because of initial misalignment of fibers in the laminate manufactured [5-6]. The most successful models up to date are based on the investigation of the stability of representative volumes where initial misalignments are present and the equilibrium equation of a fiber is imposed on its displaced configuration. Following that line of reasoning, Gutkin et al. [7] proposed a failure criterion to determine the onset of kink-band formation. All these earlier models, however, neglect damage propagation and lack therefore the ability to predict ultimate failure loads [8]. Moreover, they do not rely on or propose specially formulated finite elements that possess the capabilities to describe damage propagation in ways similar to those observed for instance in decohesive type elements. More recently, a number of published works included damage propagation modeling in longitudinal crushing of composites [9-10], but they do not explicitly consider microbuckling as the main damage mechanism. This work proposes to unite a microbuckling explicit model with matrix crack damage propagation resulting in a new alternative way to investigate failure under longitudinal compression.

\section{Representative volume and matrix shear strain}

The fibers are assumed to be cylindrical with diameter $\phi_{f}$. In a $3 \mathrm{D}$ situation fibers are arranged in hexagonal packing with a fiber volume fraction $v_{f}$. Figure 1 presents the $3 \mathrm{D}$ and $2 \mathrm{D}$ configurations. The total area $A$ of the hexagon in Fig. 1 is $A$ $=3 \sqrt{ } 3\left(t_{m}+\phi_{f}\right)^{2} / 2$ and the area covered by fibers is $A_{f}=$ 
$3 \pi \phi_{f}^{2} / 4$. Hence, the fiber volume fraction is $v_{f}=A_{f} / A$ leading to the conclusion that

$$
t_{m}=\phi_{f}\left(\sqrt{\frac{\pi}{2 \sqrt{3} v_{f}}}-1\right)
$$
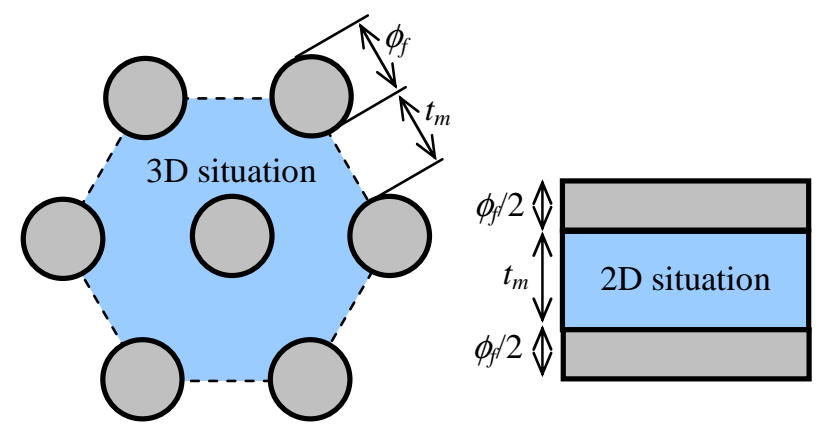

Figure 1: representative volumes

whereas a 2D model implies

$$
v_{f}^{2 D}=\frac{\phi_{f}}{\phi_{f}+t_{m}}
$$

The representative volume of the composite ply in the kinkband region is shown in Fig. 2, where the unloaded and loaded configurations are sketched. The initial geometric configuration of the representative volume is completely described by its length $\delta x$, the initial fiber misalignment angle $\theta_{0}$ and the width of the matrix layers between fibers $t_{m}$.

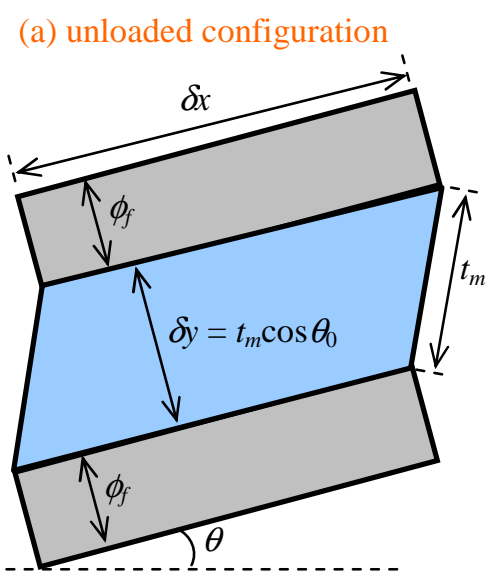

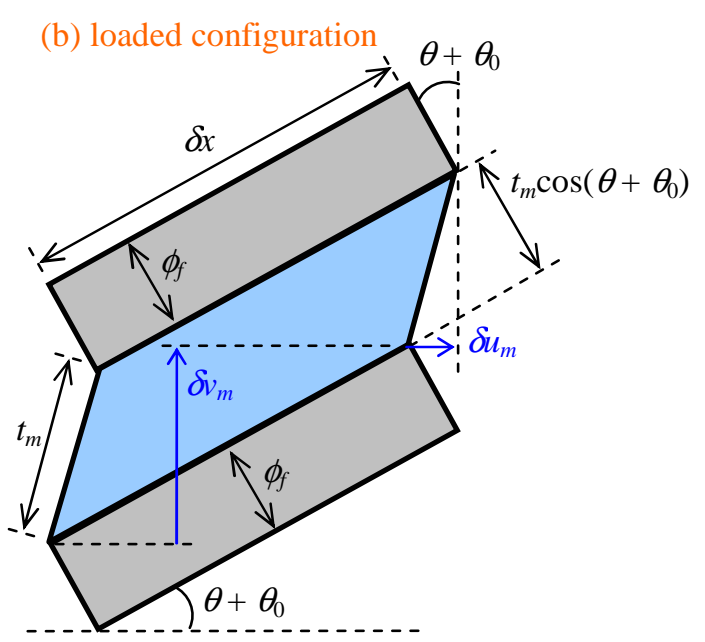

Figure 2: kink-band region: (a) unloaded and (b) loaded

The relevant geometric and kinematic information are displayed in Fig. 2. After loading the misalignment angle increases to $\theta+\theta_{0}$. It is assumed that the fibers are incompressible such that, initially, two neighboring fibers are separated by a distance $t_{m} \cos \theta_{0}$, and, after loading, this distance drops to $t_{m} \cos \left(\theta+\theta_{0}\right)$. Moreover, the length $\delta x$ is fixed. The shear deformation in the matrix is given by

$$
\gamma_{m}=\frac{\delta u_{m}}{\delta y}+\frac{\delta v_{m}}{\delta x}
$$

where $\delta u_{m}$ and $\delta v_{m}$, according to Fig. 2, can be expressed as

$$
\begin{aligned}
& \delta u_{m}=\phi_{f}\left[\sin \left(\theta+\theta_{0}\right)-\sin \theta_{0}\right] \\
& \delta_{m}=\delta x\left[\sin \left(\theta+\theta_{0}\right)-\sin \theta_{0}\right]
\end{aligned}
$$

The value of $\delta y$ can be computed as shown in Fig. 2: $\delta y=$ $t_{m} \cos \theta_{0}$. Substitution of $\delta y$ and Eq. (4) into Eq. (3) yields

$$
\begin{aligned}
\gamma_{m}= & \frac{\phi_{f}\left[\sin \left(\theta+\theta_{0}\right)-\sin \theta_{0}\right]}{t_{m} \cos \theta_{0}}+\frac{\delta x\left[\sin \left(\theta+\theta_{0}\right)-\sin \theta_{0}\right]}{\delta x}= \\
& \left(1+\frac{\phi_{f}}{t_{m} \cos \theta_{0}}\right)\left[\sin \left(\theta+\theta_{0}\right)-\sin \theta_{0}\right]
\end{aligned}
$$

Since the initial misalignment angle $\theta_{0}$ is usually small $\left(0^{\circ}<\right.$ $\theta_{0}<4^{\circ}$ ) a good approximation for Eq. (5) is

$$
\gamma_{m} \approx\left(1+\frac{\phi_{f}}{t_{m}}\right) \sin \theta
$$

It is possible to go even further assuming that $\theta$ is small and, considering Eq. (2), write

$$
\gamma_{m} \approx\left(1+\frac{\phi_{f}}{t_{m}}\right) \theta=\frac{\theta}{1-v_{f}^{2 D}}
$$

This approach linearizes the problem in the variable $\theta$, what can sometimes be useful and may provide insight into the 
constitutive relations involved. For a 2D model the shear modulus of the matrix is

$$
G_{m}^{2 D}=\frac{G_{m}}{1-v_{f}^{2 D}}
$$

Thus, provided the deformation regime within the matrix remains elastic and angle $\theta$ remains small, one can write

$$
\tau_{m}=G_{m} \gamma_{m}=\left(1-v_{f}^{2 D}\right) G_{m}^{2 D} \frac{\theta}{1-v_{f}^{2 D}}=G_{m}^{2 D} \theta
$$

\section{Equilibrium equation in the loaded configuration}

When it comes to kink-band formation the relevant homogenized stresses acting on the representative volume are $\sigma_{1}$ and $\tau_{12}$, which are depicted in Fig. 3. The fiber is subject to normal stress $\sigma_{f}$. Assuming that the fiber is much stiffer than the matrix, the classical theory of micromechanics states that

$$
\sigma_{f} \phi_{f}=\sigma_{1}\left(\phi_{f}+t_{m}\right)
$$

The classical theory of micromechanics also indicates that the shear stress is the same throughout the representative volume. Hence, the shear stress in the fiber $\tau_{f}$ is equal to the homogenized shear stress $\tau_{12}$ i.e.,

$$
\tau_{f}=\tau_{12}
$$

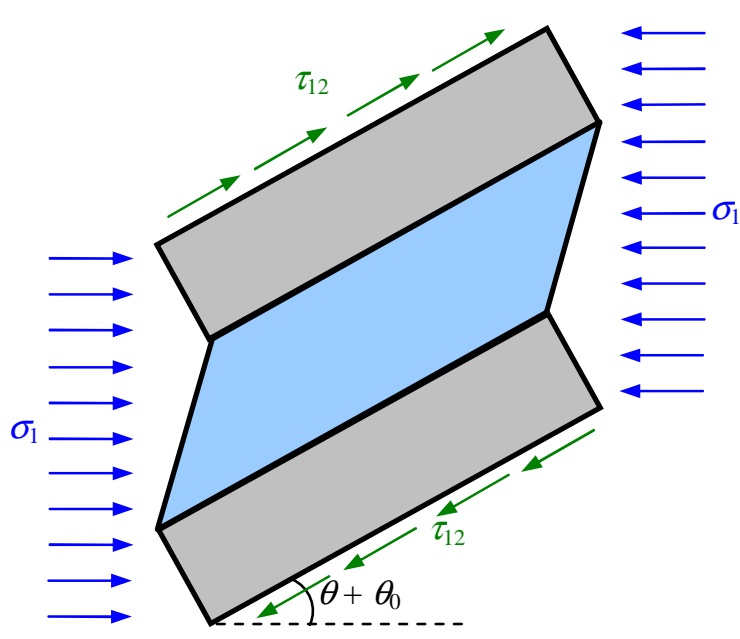

Figure 3: Homogenized stresses applied to the representative volume

The free body diagram of a single fiber in the loaded configuration is shown in Fig. 4. The fiber orientation in the unloaded configuration is also shown with the initial misalignment angle $\theta_{0}$. Force $P$ is the net effect of the normal stresses in the fiber $\sigma_{f}$ and force $S$ is the net effect of the shear stresses in the fiber $\tau_{f}$ such that, with the aid of Eqs. (10) and (11),

$$
P=\sigma_{f} \phi_{f}=\sigma_{1}\left(\phi_{f}+t_{m}\right) \quad, \quad S=\tau_{f} \phi_{f}=\tau_{12} \phi_{f}
$$

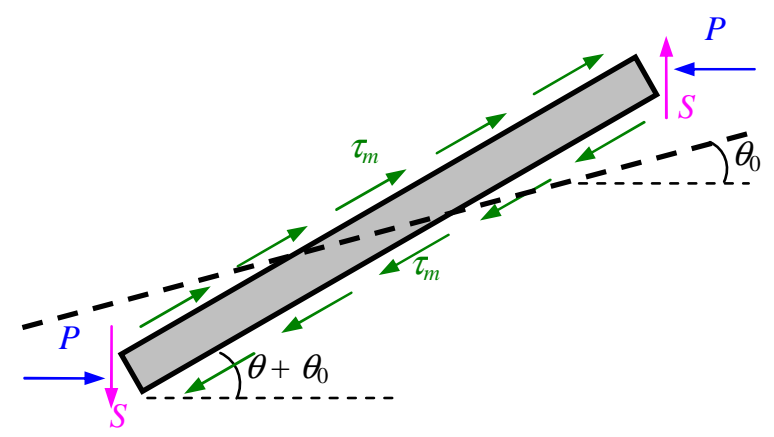

Figure 4: Free body diagram of fiber in the loaded configuration

The force equilibrium is clearly satisfied. Imposition of moment equilibrium yields

$$
P \delta x \sin \left(\theta+\theta_{0}\right)+S \delta x \cos \left(\theta+\theta_{0}\right)=\tau_{m} \phi_{f} \delta x
$$

Substitution of Eq. (2) and (12) in (13) leads to

$$
\frac{\sigma_{1}}{v_{f}^{2 D}} \sin \left(\theta+\theta_{0}\right)+\tau_{12} \cos \left(\theta+\theta_{0}\right)=\tau_{m}
$$

Failure of the fiber is assumed to happen abruptly when the fiber stress $\sigma_{f}$ in Eq. (12) reaches the fiber compressive strength $X_{f}^{c}$. Before reaching $X_{f}^{c}$ it is assumed that the fibers behave linearly.

Equation (14) describes the buckling of an initially displaced rigid bar under the action of compressive and shear forces as shown in Fig. 5. If the applied forces $P, S$ and the spring constant $k$ are given by

$$
P=\frac{\sigma_{1}}{L v_{f}^{2 D}} \quad, \quad S=\frac{\tau_{12}}{L} \quad, \quad k=\frac{G_{m}^{2 D}}{L}
$$

the moment equilibrium equation matches exactly Eq. (14), provided $\tau_{m}$ is given by Eq. (9). Even if Eq. (9) is not applicable, the original relation $\tau_{m}=G_{m} \gamma_{m}$ can still be used and the spring constant $k$ may be assumed to vary with $\theta$.

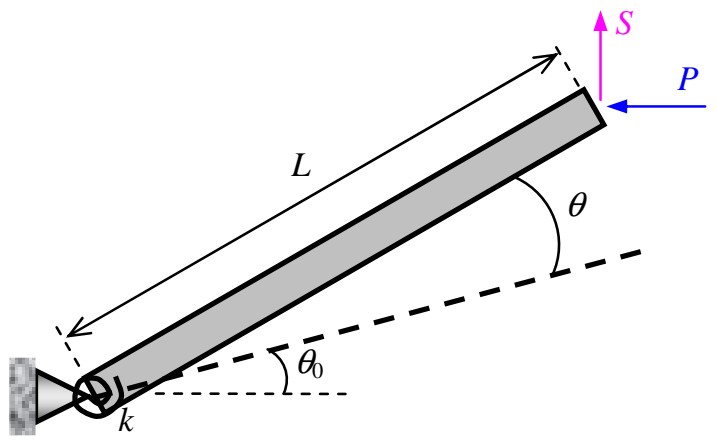

Figure 5: Initially displaced rigid bar

Notice that, if one assumes elastic regime and small angles, then substitution of Eq. (9) into Eq. (14) will result in a linear equation that can be solved for $\theta$. Hence, under these simplifying assumptions, the moment equilibrium equation 
can be explicitly solved for $\theta$. A simple stress based criterion for fiber kinking can be subsequently proposed if energy balance considerations during crack formation come into play. This, however, is not the objective of this work. Instead, a model that goes beyond the onset of failure shall be proposed.

\section{The matrix cohesive model}

The proposed model behavior of the matrix in shear is shown in Fig. 6. The damage parameter starts at zero and reaches the maximum value 1 when the failure shear strain $\gamma_{m f}$ is reached and, consequently, the matrix has no stiffness left, becoming completely degraded (see Fig. 6). $S_{L}$ is a matrix property that determines the onset of matrix failure.

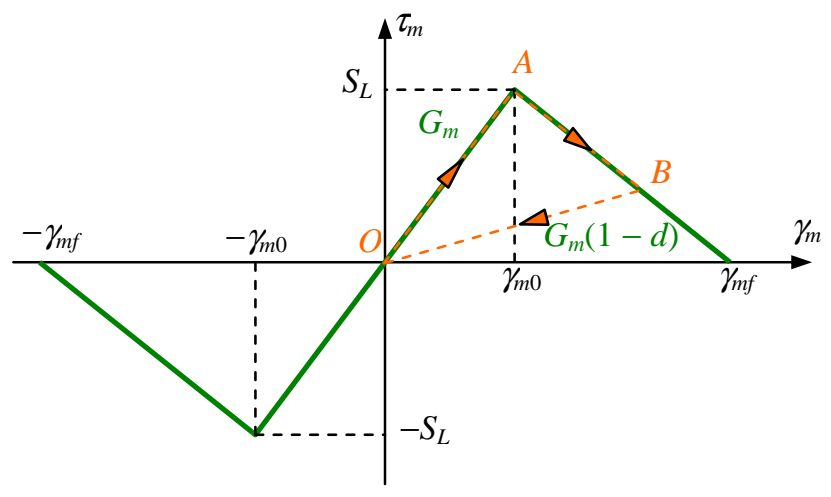

Figure 6: Model behavior in shear

Once the initiation $\left(\gamma_{m 0}\right)$ and failure $\left(\gamma_{m f}\right)$ shear strains are available, the damage parameter can be expressed as

$$
d=\frac{\gamma_{m f}}{\gamma_{m f}-\gamma_{m 0}}\left(1-\frac{\gamma_{m 0}}{\gamma_{m}}\right)
$$

Equation (16) is valid only for strain values that obey $\left|\gamma_{m}\right|>$ $\gamma_{m 0}$, otherwise $d=0$. If the matrix becomes damaged then $d$ $>0$ and $d$ never decreases, i.e., the model does not allow for restoration of mechanical properties once the matrix is damaged. Take for instance the loading path $O A B$ in Fig. 6. If, after returning to point $O$ the material is again loaded, it will follow path $O B$ until it reaches point $B$. If further loading is applied beyond point $B$ the original curve of the constitutive law is retaken until $d=1$, when total failure is reached.

Figure 6 shows that the inclination of the $O B$ path is $G_{m}(1-$ $d)$. Hence, generally the stress $\times$ strain relation can be written as

$$
\tau_{m}=G_{m}(1-d) \gamma_{m}
$$

which, in the incremental form reads

$$
\Delta \tau_{m}=G_{m}(1-d) \Delta \gamma_{m}-G_{m} \gamma_{m} \Delta d
$$

In Eq. (18) it is tacitly assumed that there is no plastic shear strain.

\section{Incremental model and the finite element context}

Two parameters are important to describe kink-band formation: $\theta$ that can be computed from Eq. (14) and $d$ that can be computed from Eq. (16). It is also assumed that the fibers respond linearly up to catastrophic failure when $\sigma_{f}=$ $X_{f}^{c}$. Thus, the constitutive relations for both fiber and matrix are

$$
\sigma_{f}=E_{f} \varepsilon_{f} \quad, \quad \tau_{f}=G_{f} \gamma_{f} \quad, \quad \sigma_{m}=E_{m} \varepsilon_{m} \quad, \quad \tau_{m}=G_{m} \gamma_{m}
$$

where $E_{f}$ and $G_{f}$ are assumed to be constant throughout. The third equation $\sigma_{m}=E_{m} \varepsilon_{m}$ may pose a problem since the matrix elastic modulus $E_{m}$ is needed and it would clearly vary depending on the degree of degradation of the matrix. One possible way around this difficulty is to adopt a damage propagation model for the matrix under traction/compression similar to the one proposed in Fig. 6 for the matrix under shear. This would result in one additional damage parameter. Another option is to consider that, since kink-band formation is the relevant failure mechanism in this study, the matrix will be under compressive stresses just like the fibers. Hence, equation $\sigma_{m}$ $=E_{m} \varepsilon_{m}$ can be used up to the point where $\sigma_{m}$ is equal to the matrix strength under compression $X_{m}{ }^{c}$. In this study the later option is considered.

The in-plane mechanical properties of a lamina can be computed using the Halpin-Tsai rules of mixture as

$$
\begin{gathered}
E_{1}=E_{f} v_{f}^{2 D}+E_{m}\left(1-v_{f}^{2 D}\right) \\
v_{12}=v_{f} v_{f}^{2 D}+v_{m}\left(1-v_{f}^{2 D}\right) \\
\xi=1+40\left(v_{f}^{2 D}\right)^{10} \\
\eta_{E}=\frac{\left(E_{f} / E_{m}\right)-1}{\left(E_{f} / E_{m}\right)+\xi} \\
E_{2}=E_{m} \frac{1+\xi \eta_{E} v_{f}^{2 D}}{1-\eta_{E} v_{f}^{2 D}} \\
\eta_{G}=\frac{\left(G_{f} / G_{m}\right)-1}{\left(G_{f} / G_{m}\right)+\xi} \\
G_{12}=G_{m} \frac{1+\xi \eta_{G} v_{f}^{2 D}}{1-\eta_{G} v_{f}^{2 D}}
\end{gathered}
$$

where $v_{f}$ and $v_{m}$ are the fiber and matrix Poisson ratios, respectively, assumed constant. The ply constitutive equation is

$$
\left\{\begin{array}{l}
\sigma_{1} \\
\sigma_{2} \\
\tau_{12}
\end{array}\right\}=\frac{1}{1-v_{12} \nu_{21}}\left[\begin{array}{ccc}
E_{1} & v_{12} E_{2} & 0 \\
v_{21} E_{1} & E_{2} & 0 \\
0 & 0 & \left(1-v_{12} \nu_{21}\right) G_{12}
\end{array}\right]\left\{\begin{array}{l}
\varepsilon_{1} \\
\varepsilon_{2} \\
\gamma_{12}
\end{array}\right\}
$$

which, in the incremental form reads 


$$
\left\{\begin{array}{c}
\Delta \sigma_{1} \\
\Delta \sigma_{2} \\
\Delta \tau_{12}
\end{array}\right\}=\frac{1}{1-v_{12} \nu_{21}}\left[\begin{array}{ccc}
E_{1} & v_{12} E_{2} & 0 \\
v_{21} E_{1} & E_{2} & 0 \\
0 & 0 & \left(1-v_{12} \nu_{21}\right) G_{12}
\end{array}\right]\left\{\begin{array}{c}
\Delta \varepsilon_{1} \\
\Delta \varepsilon_{2} \\
\Delta \gamma_{12}
\end{array}\right\}+\left\{\begin{array}{c}
0 \\
0 \\
\gamma_{12} \Delta G_{12}
\end{array}\right\}
$$

Notice that the matrix shear modulus $G_{m}$ influences only $G_{12}$ in Eq. (26). Hence, the term $\gamma_{12} \Delta G_{12}$ must be accounted for in Eq. (28). The other ply mechanical properties $\left(E_{1}, E_{2}, v_{12}\right)$ are assumed to be independent of $G_{m}$.

In the finite element context the problem must be solved incrementally. Initially consider that the equilibrium problem in the weak form has been solved for time step $n$ such that

$$
\sum_{e=1}^{N_{e}} \int_{V_{e}} \delta \mathbf{q}_{e}^{T} \mathbf{B}_{e}^{T} \boldsymbol{\sigma}_{e}^{n} d V_{e}=\sum_{e=1}^{N_{e}} \int_{V_{e}} \delta \mathbf{q}_{e}^{T} \mathbf{f}_{e}^{n} d V_{e}
$$

where summation extends over all $N_{e}$ elements in the mesh and $\mathbf{f}_{e}$ is the vector of external loads applied to element $e$. Matrix $\mathbf{B}_{e}$ is the discretization matrix that relates strains within a given element to the element degrees of freedom stored in $\mathbf{q}_{e}: \boldsymbol{\varepsilon}_{e}=\mathbf{B}_{e} \mathbf{q}_{e}$. At the subsequent time step $n+1$ the new equilibrium equation in the weak form reads

$$
\sum_{e=1}^{N_{e}} \int_{V_{e}} \delta \mathbf{q}_{e}^{T} \mathbf{B}_{e}^{T} \boldsymbol{\sigma}_{e}^{n+1} d V_{e}=\sum_{e=1}^{N_{e}} \int_{V_{e}} \delta \mathbf{q}_{e}^{T} \mathbf{f}_{e}^{n+1} d V_{e}
$$

with $\boldsymbol{\sigma}_{e}^{n+1}=\boldsymbol{\sigma}_{e}^{n}+\Delta \boldsymbol{\sigma}_{e}^{n+1}$. Subtraction of Eq. (29) from Eq. (30), and recognizing that $\delta \mathbf{q}_{e}$ are arbitrary and geometrically admissible virtual displacements

$$
\sum_{e=1}^{N_{e}} \int_{V_{e}} \mathbf{B}_{e}^{T} \Delta \boldsymbol{\sigma}_{e}^{n+1} d V_{e}=\mathbf{f}_{e x t}^{n+1}-\mathbf{f}_{e x t}^{n}=\Delta \mathbf{f}_{e x t}
$$

where the combined effects of all $\mathbf{f}_{e}$ have been grouped into $\mathbf{f}_{e x t}$. Solution to Eq. (31) must be found iteratively. All the terms relating to $\Delta \boldsymbol{\sigma}_{e}^{n+1}$ in the left-hand side of Eq. (31) must be computed at the Gaussian stations used in the numerical integration.

Equations (5) and (14) in the incremental forms read

$$
\begin{gathered}
\Delta \gamma_{m}=\left(1+\frac{\phi_{f}}{t_{m} \cos \theta_{0}}\right) \Delta \theta \cos \left(\theta+\theta_{0}\right) \\
\frac{\Delta \sigma_{1}}{v_{f}^{2 D}} \sin \left(\theta+\theta_{0}\right)+\Delta \tau_{12} \cos \left(\theta+\theta_{0}\right)+ \\
\Delta \theta \frac{\sigma_{1}}{v_{f}^{2 D}} \cos \left(\theta+\theta_{0}\right)-\Delta \theta \tau_{12} \sin \left(\theta+\theta_{0}\right)=\Delta \tau_{m}
\end{gathered}
$$

Substitution of Eqs. (5), (18) and (32) into (33) yields

$$
\begin{aligned}
& \frac{\Delta \sigma_{1}}{v_{f}^{2 D}} \sin \left(\theta+\theta_{0}\right)+\Delta \tau_{12} \cos \left(\theta+\theta_{0}\right)+ \\
& \Delta \theta \frac{\sigma_{1}}{v_{f}^{2 D}} \cos \left(\theta+\theta_{0}\right)-\Delta \theta \tau_{12} \sin \left(\theta+\theta_{0}\right)= \\
& G_{m}(1-d)\left(1+\frac{\phi_{f}}{t_{m} \cos \theta_{0}}\right) \Delta \theta \cos \left(\theta+\theta_{0}\right)- \\
& G_{m}\left(1+\frac{\phi_{f}}{t_{m} \cos \theta_{0}}\right)\left[\sin \left(\theta+\theta_{0}\right)-\sin \theta_{0}\right] \Delta d
\end{aligned}
$$

Therefore, given the stress and damage increments $\left(\Delta \sigma_{1}\right.$, $\left.\Delta \tau_{12}, \Delta d\right), \Delta \theta$ can be computed and the fiber misalignment is updated to $\theta+\Delta \theta$.

\section{Numerical results}

A $1 \mathrm{~m} \times 1 \mathrm{~m} \times 0.125 \mathrm{~mm}$ single layer specimen, with fibers oriented along the $x$ axis is subject to compressive prescribed displacement also along $x$. Figure 7 depicts the situation. Only the center node is restricted to move along the $y$ direction in order to avoid rigid body mode. A $10 \times 10$ mesh of bilinear elements is used.

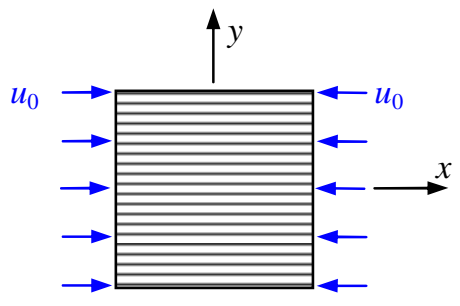

Figure 7: Single layer specimen under compression

The material properties used are reported in Table 1 . Notice that the initiation $\left(\gamma_{m 0}\right)$ and failure $\left(\gamma_{m f}\right)$ shear strains of the fibers are unnecessary for the present model. The fiber volume fraction is assumed to be $60 \%$.

Table 1: Material properties used

\begin{tabular}{ccc}
\hline property & matrix & fiber \\
\hline$E_{11}[\mathrm{GPa}]$ & 2.6 & 240 \\
$E_{22}[\mathrm{GPa}]$ & 2.6 & 240 \\
$v_{12}$ & 0.38 & 0.22 \\
$G_{12}[\mathrm{GPa}]$ & 0.94 & 98.36 \\
$\gamma_{m 0}$ & 0.002 & - \\
$\gamma_{m f}$ & 0.005 & - \\
\hline
\end{tabular}

The initial fiber misalignment $\theta_{0}$ is defined (in degrees) according to Eq. (35)

$$
\theta_{0}=3.5^{\circ} \cos (\pi \alpha / L)
$$

where $L$ is the edge length $(L=1 \mathrm{~m})$ and $-L / 2 \leq x \leq L / 2$. 
Figure 8 presents the result in terms of damage parameter $d$ computed with Eq. (16). Figure 8 is obtained for the ultimate displacement $u_{0}=6 \mathrm{~mm}$ as illustrated in Fig. 7 .

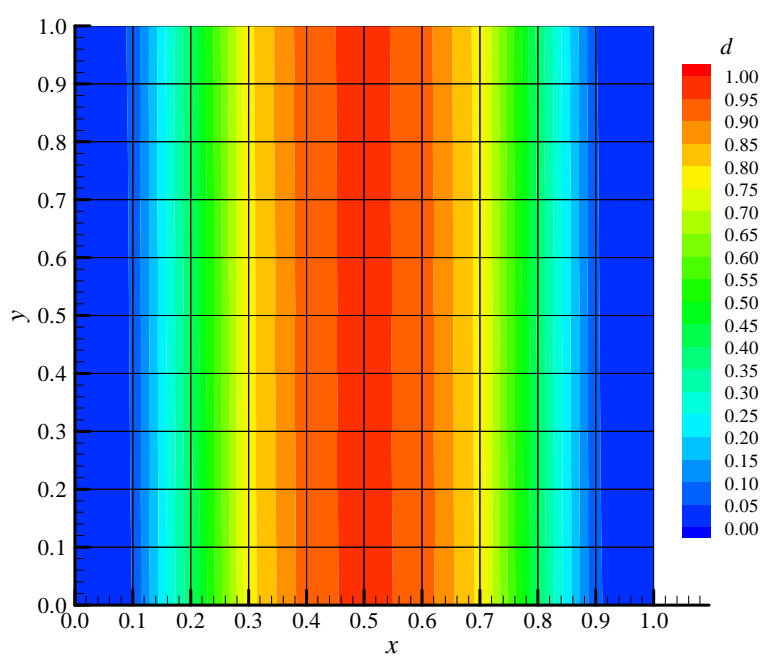

Figure 8: Damage parameter mapping

Figure 9 presents the evolution of the matrix shear stress $\tau_{m}$ and damage parameter $d$ computed at the center node of the mesh. Observe that the $\tau_{m}$ behavior is consistent with Fig. 6 and the red curve for $d$ follows the hyperbolic pattern described in Eq. (16).

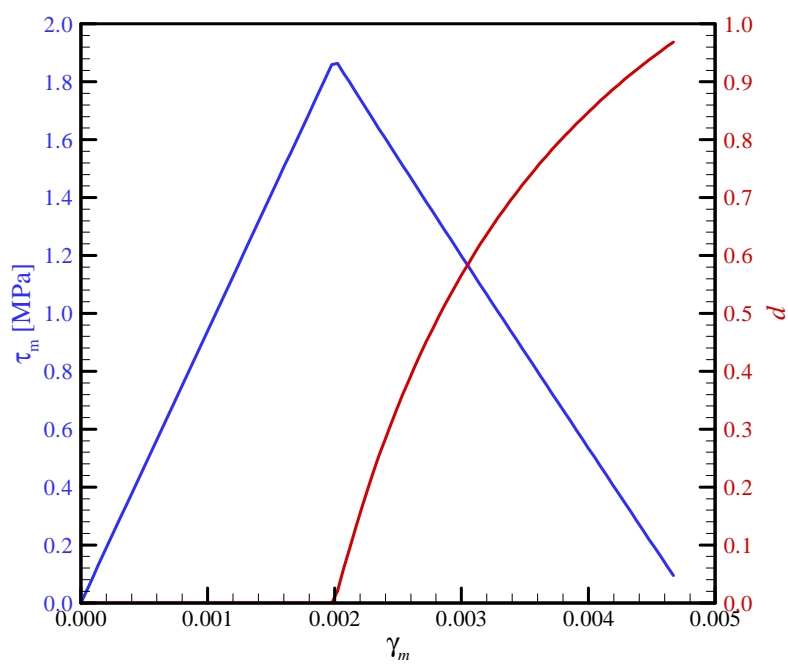

Figure 9: Matrix shear stress and damage parameter evolution

\section{Conclusions}

A model is proposed to capture the onset of formation of kink-bands and subsequently its propagation. It is formulated within the context of an energy based approach that considers the critical energy release rate of the matrix in the failed elements of the mesh. A damage parameter that ranges from 0 (intact) to 1 (fully damaged) is used that relates relative shear strains in the matrix induced by loss of stability of a representative volume.

Today commercially available finite element packages (e.g. Abaqus) possess damage modeling capabilites. However, they are not well suited for predictions of failure under longitudinal compression since they dot not inherently consider microbuckling and kink band formation, therefore delivering poor results whenever crushing is involved. The present model may be coupled with additional models for damage propagation and failure predictions [11], and latter incorporated into a commercial FE code. An element deletion criterion would then be required in order to simulate more realistic composite structures.

The model is based on physical principles and requires only two parameters whose extraction is not difficult: the initiation $\left(\gamma_{m 0}\right)$ and failure $\left(\gamma_{m f}\right)$ shear strains of the matrix. The numerical result present shows that the model is simple to implement and captures the most essential behavior of structural failure under compressive loadings.

\section{References}

[1] Rosen BW. Mechanics of composite strengthening. Fibre composite materials. Metals Park, Ohio: American Society of Materials; 1965.

[2] Argon AS. Fracture of composites. Treatise on materials science and technology. New York: Academy Press; 1972.

[3] Steif PS. A model for kinking in fiber composites I. fiber breakage via micro-buckling. International Journal of Solids and Structures 26(5/6): 549-561, 1990.

[4] Steif PS. A model for kinking in fiber composites II. kink band formation. International Journal of Solids and Structures 26(5/6): 563-569, 1990.

[5] Pimenta S, Gutkin R, Pinho ST, Robinson P. A micromechanical model for kink-band formation: Part I - experimental study and numerical modelling. Composites Science and Technology 69: 948-955, 2009.

[6] Pimenta S, Gutkin R, Pinho ST, Robinson P. A micromechanical model for kink-band formation: Part II - analytical modelling. Composites Science and Technology 69: 956-964, 2009.

[7] Gutkin R, Pinho ST, Robinson P, Curtis PT. A finite fracture mechanics formulation to predict fibre kinking and splitting in CFRP under combined longitudinal compression and in-plane shear. Mechanics of Materials 43: 730-739, 2011.

[8] Dávila CG, Camanho PP. Failure criteria for FRP laminates in plane stress. NASA/TM-2003212663, 2003.

[9] Gutkin R, Pinho ST. Combining damage and friction to model compressive damage growth in 
fibre-reinforced composites. Journal of Composite Materials 49(20): 2483-2495, 2015.

[10] Gutkin R, Costa S, Olsson R. A physically based model for kink-band growth and logitudinal crushing of composites under 3D stress states accounting for friction. Composites Science and Technology 135: 39-45, 2016.

[11] Bürger D, de Faria AR, Almeida SFM, Melo FCL, Donadon MV. Ballistic impact simulation of an armour-piercing projectile on hybrid ceramic/fiber reinforced composite armours. International Journal of Impact Engineering, 43: 63-7, 2012. 